Cristina Mendes Giglotti Borsarl

Rosel Mieko Yamamoto Nomura ${ }^{1}$

Gláucia Rosana Guerra Benute ${ }^{2}$

Mara Cristina Souza de Lucia ${ }^{3}$

Rossana Pulcinel Viera Francisco ${ }^{1}$

MARCElo Zugaib ${ }^{1}$

Artigo Original

Palavras-chave

Aborto

Aborto induzido

Aborto espontâneo

Saúde da mulher

Fatores socioeconômicos

Keywords

Abortion

Abortion, induced Spontaneous abortion

Women's health

Socioeconomic factors

\title{
Aborto provocado em mulheres da periferia da cidade de São Paulo: vivência e aspectos socioeconômicos
}

\author{
Abortion in women living in the outskirts of Sao Paulo: experience \\ and socioeconomic aspects
}

\section{Resumo}

OBJETIVO: Comparar e analisar aspectos socioeconômicos e emocionais na vivência do aborto provocado e espontâneo em mulheres da periferia da cidade de São Paulo. MÉTODOS: Estudo prospectivo e caso-controle realizado no período de julho de 2008 a março de 2010, envolvendo a realização de entrevistas semidirigidas, previamente elaboradas com mulheres que apresentavam diagnóstico médico de aborto internadas em dois hospitais públicos da periferia da cidade de São Paulo. Foram incluídas 100 mulheres com diagnóstico de aborto que foram internadas para a realização da curetagem uterina. Foram identificadas 11 mulheres que relataram ter provocado aborto (1 1\%) que constituíram o grupo de casos. $\bigcirc$ grupo controle (n=22) foi selecionado na proporção 2:1, seguindo-se o procedimento: para cada caso de aborto provocado, os próximos dois casos de aborto espontâneo, do mesmo hospital. Foi realizada entrevista semiestruturada com perguntas relativas aos aspectos emocionais, ao contexto familiar, social e econômico. RESULTADOS: As mulheres do grupo com aborto provocado, em relação ao grupo com aborto espontâneo, apresentaram menor escolaridade, sendo mais frequente o nível fundamental (82 versus 36\%, $\mathrm{p}=0,04$ ); menor renda familiar (mediana, $\mathrm{R} \$ 1.000,00$ versus $\mathrm{R} \$ 1.400,00, \mathrm{p}=0,04$ ); menor renda pessoal (mediana, $R \$ 200,00$ versus $R \$ 333,00, p=0,04$ ), maior frequência de sentimentos negativos na suspeita (82 versus $22 \%, p=0,004)$ e na confirmação $(72$ versus $22 \%, p=0,03)$ da gravidez. CONCLUSÃO: $\bigcirc$ aborto provocado em mulheres que procuram atendimento em hospitais da periferia da cidade de São Paulo está relacionado a condições socioeconômicas desfavoráveis, o que prejudica a vivência na suspeita e confirmação da gravidez.

\section{Abstract}

PURPOSE: To compare and analyze socioeconomic aspects and the emotional experience of women with spontaneous or induced abortion and in women living in the outskirts of São Paulo. METHODS: A prospective case-control study carried out from July 2008 to March 2010, involving semi-structured interviews with women who presented a previous diagnosis of abortion and who had been admitted to two public hospitals in the outskirts of São Paulo. The study included 100 women with diagnosis of abortion and were hospitalized for curettage. Eleven women who reported induced abortion (1 1\%) represented the case group. The control group ( $n=22$ ) was selected at a 2:1 ratio according to the following procedure: for every case of induced abortion, the next two cases of spontaneous abortion at the same hospital. A semistructured interview was conducted with questions regarding emotional aspects and family, social and economic context. RESULTS: The women with induced abortion compared to the group with spontaneous abortion had lower educational level, with more frequent elementary level (82 versus 36\%, $\mathrm{p}=0.04$ ), lower income (median, $\mathrm{R} \$ 1,000.00$ versus $R \$ 1,400.00, p=0.04$ ), lower personal income (median, $R \$ 200.00$ versus $R \$ 333.00, p=0.04$ ), higher frequency of negative feelings upon suspicion ( 82 versus $22 \%, p=0.004$ ) and confirmation (72 versus $22 \%, p=0.03$ ) of pregnancy. CONCLUSION: Among women looking for health care in hospitals in the outskirts of São Paulo, induced abortion is related to unfavorable socioeconomic conditions, which affects the emotional experiences of suspicion and confirmation of pregnancy.

Correspondêncic

Roseli Mieko Yamamoto Nomura Departamento de Obstetríicia e Ginecologia, Faculdade de Medicina da Universidade de São Paulo Av. Dr. Enéas de Carvalho Aguiar 255 - $10^{\circ}$ andar - sala 10037 (EP: 05403-000

São Paulo (SP), Brasi
'Disciplina de Obstetrícia do Departamento de Obstetrícia e Ginecologia da Faculdade de Medicina, Universidade de São Paulo USP - São Paulo (SP), Brasil.

${ }^{2}$ Serviço de Saúde da Divisão de Psicologia do Instituto Central do Hospital das Clínicas da Faculdade de Medicina, Universidade de São Paulo - USP - São Paulo (SP), Brasil.

${ }^{3}$ Divisão de Psicologia do Instituto Central do Hospital das Clínicas da Faculdade de Medicina, Universidade de São Paulo - USP São Paulo (SP), Brasil. 
Introdução

O aborto é um tema ainda muito estigmatizado, seja pelos aspectos morais, religiosos e éticos ou por ser assunto marginalizado em nossa sociedade. Fato é que este tema carrega grande complexidade em suas múltiplas faces, pois a mulher que sofre ou provoca aborto vivencia ambivalência de sentimentos e, muitas vezes, negligencia sua própria condição.

A Organização Mundial de Saúde (OMS) define o aborto como a interrupção da gravidez antes da viabilidade do produto da concepção, correspondendo à perda do concepto até a $20^{\mathrm{a}}$ e a $22^{\mathrm{a}}$ semanas completas e/ou com feto de até $500 \mathrm{~g}^{1}$. É considerado espontâneo quando se inicia independentemente de qualquer procedimento ou mecanismo externo, geralmente devido a problemas de saúde da mulher ou do feto. É considerado provocado quando resulta da utilização de qualquer processo abortivo externo, químico ou mecânico. Este pode ter motivação voluntária ou involuntária da gestante, e ser considerado legal ou ilegal ${ }^{2}$.

No Brasil, o aborto ainda se destaca como grave problema de saúde pública, na medida em que é amplamente praticado, muitas vezes de forma insegura, dentro de um cenário de clandestinidade ${ }^{3}$. A curetagem pós-abortamento representa o segundo procedimento mais realizado nas unidades de internação da rede pública de serviços de saúde, superada apenas pelos partos normais ${ }^{4}$. Dados do Ministério da Saúde, do Sistema de Informações Hospitalares do Sistema Único de Saúde (SUS), revelam registro anual, a partir de 1999, de em média 238.000 internações para realização de curetagem por diagnóstico de aborto 5 . Portanto, a ilegalidade do aborto não coíbe a prática e perpetua as iniquidades socioeconômicas em que é realizado. Há, assim, um contexto que impõe às mulheres oportunidades desiguais de evitar uma gravidez ou de escolher seu desfecho ${ }^{6}$. São especialmente as mulheres em condições socioeconômicas menos favorecidas aquelas que se submetem aos riscos da prática do aborto realizado em condições precárias ${ }^{7}$.

No Brasil ocorrem aproximadamente um milhão e quinhentos mil abortos espontâneos e inseguros com taxa de 3,7 para cada 100 mulheres $^{8}$, e $4 \%$ das mulheres em idade fértil recorrem ao aborto provocado no período de um ano?.

Estudos apontam que a realização do aborto é experiência que traz consequências físicas e emocionais ruins para as mulheres, e que a decisão de fazê-lo não é inconsequen$\mathrm{te}^{10-12}$. O aborto é problema sério para as mulheres, mas também para a sociedade que, de certa forma, influencia sua realização e acaba sofrendo as consequências em termos de saúde pública e de conflitos sociais ${ }^{13}$.

A ilegalidade do aborto faz com que sua real magnitude seja desconhecida, e suas causas e consequências para a saúde das mulheres, obscurecidas. Dentro dessa perspectiva, o presente artigo se propõe a apresentar uma comparação entre as mulheres que sofrem aborto espontâneo e as que o provocam em um extrato socioeconômico menos favorecido da periferia da cidade de São Paulo, e também comparar como essas mulheres vivenciam emocionalmente esta experiência.

\section{Métodos}

Este estudo prospectivo, caso-controle e transversal foi desenvolvido na Disciplina de Obstetrícia do Departamento de Obstetrícia e Ginecologia da Faculdade de Medicina da Universidade de São Paulo, no período entre julho de 2008 e março de 2010 . O protocolo de pesquisa e o termo de consentimento livre e esclarecido foram aprovados pelo Comitê de Ética em Pesquisa da instituição.

Foram realizadas entrevistas semiestruturadas com roteiro, previamente aplicadas em dois hospitais públicos da periferia da cidade de São Paulo: Hospital Estadual Sapopemba, no período de julho a dezembro de 2008; e no Hospital Municipal Dr. Moysés Deustch - M' Boi Mirim no período de março de 2009 a março de 2010. As entrevistas foram realizadas por uma única pesquisadora, psicóloga, e estas instituições foram escolhidas por apresentarem a população-alvo (mulheres em um contexto socioeconômico menos favorecido) e por ser o local de trabalho da pesquisadora. As mulheres que concordaram em participar deste estudo assinaram, antes do início da entrevista, o termo de consentimento livre e esclarecido, no qual constavam informações sobre os objetivos da pesquisa e esclarecimento da confidencialidade dos dados informados.

Foram incluídas 100 mulheres que apresentavam o diagnóstico médico de aborto, e foram internadas para realização do procedimento de curetagem uterina. Os critérios de exclusão foram: mulheres que se negaram a serem entrevistadas, adolescentes que estavam sem acompanhante legal, aquelas que apresentaram sinais de confusão mental ou incompreensão das perguntas. Assim, as mulheres que cumpriram os critérios de inclusão foram convidadas a participar da pesquisa. A abordagem da paciente era realizada respeitando o momento de sua vivência, ou seja, a mulher era convidada pela psicóloga para participar da pesquisa, esclarecendo-se a importância do tema e a necessidade de se obter mais conhecimento sobre as condições emocionais envolvidas na vivência do aborto. A psicóloga realizava busca ativa através do censo de enfermagem das mulheres internadas com diagnóstico de aborto. As pacientes sempre eram abordadas após procedimento de curetagem, no leito da enfermaria e em condições clínicas favoráveis. Este fato, de entrevistá-las apenas após o procedimento de curetagem, garantia a estas 
mulheres nenhum prejuízo ou atraso de seu tratamento. As entrevistas foram realizadas em ambiente que garantisse a privacidade das pacientes, portanto, a abordagem se iniciava na beira do leito. Contudo, consentindo em participar, a paciente era conduzida a uma sala reservada para os atendimentos psicológicos.

Pela entrevista semiestruturada foram coletados os dados de identificação e foram realizadas perguntas relativas aos aspectos emocionais, ao contexto familiar, social e econômico. O questionário foi composto de perguntas fechadas que incluíam os seguintes dados: idade (anos), escolaridade (ensino fundamental, médio ou superior), estado conjugal (com companheiro ou sem companheiro), ocupação (com ou sem atividade laboral, ou estudante), número de gestações, número de partos, número de abortos, antecedente de aborto provocado (sim ou não), número de filhos vivos, idade gestacional do aborto atual (em semanas), religião (católica, evangélica, outros e sem religião), crença de fé (presente ou ausente), renda familiar mensal (em reais), número de pessoas na família e renda per capita (em reais).

Foram formuladas questões abertas ou semidirigidas abordando os seguintes aspectos: sentimentos vividos por ocasião da suspeita sobre ocorrência de gestação e quando a mesma foi confirmada, e existência de apoio familiar, de amigos ou do parceiro frente à situação do abortamento. Foi também investigada a existência de sentimento de culpa ou arrependimento relacionado ao aborto. Com o transcorrer da entrevista, a entrevistadora assegurava o caráter sigiloso da entrevista e a garantia da confidencialidade dos dados informados, e, nessa condição, a mulher era estimulada a esclarecer sobre qual foi o tipo de abortamento, espontâneo ou provocado.

Para a análise dos sentimentos vivenciados relatados na entrevista semidirigida, foi utilizada a técnica de Análise Temática ou de Conteúdo, que tem como objetivo descrever, interpretar e compreender os dados. A técnica foi utilizada para explanar de forma objetiva, sistemática e quantitativa o conteúdo manifestado nas entrevistas. O foco não foi descrever os conteúdos, mas sim verificar o que foi revelado após o tratamento dos dados pela codificação dos mesmos, transformando-os em categorias por meio de uma análise transversal. As entrevistas foram recortadas ao redor de cada tema-eixo ou unidade de significação de interesse. Os núcleos de sentido que compõem a comunicação de cada paciente foram delineados e, então, realizados os recortes para categorizar os conteúdos. Todos os resultados obtidos com a categorização foram analisados com técnicas quantitativas.

Foram identificadas 11 mulheres que provocaram aborto (11\%), as quais constituíram o grupo de casos. O grupo controle foi selecionado na proporção 2:1, seguindo-se o procedimento: para cada caso de aborto provocado, os próximos dois casos de aborto espontâneo, do mesmo hospital, foram selecionados entre os 89 casos entrevistados de aborto espontâneo. Com isso, procurou-se ponderar a proporcionalidade de controles de acordo com o hospital envolvido na pesquisa. As características das mulheres estão apresentadas na Tabela 1.

A análise dos dados quantitativos foi realizada com o emprego do programa Medcalc (Medcalc software bvba, versão 11.5.1.0), calculando-se médias e desvios-padrão, frequências absolutas e relativas. As variáveis categóricas foram avaliadas pelo teste do $\chi^{2}$ ou teste exato de Fisher, quando indicado. As variáveis contínuas foram analisadas pelo teste $t$ de student ou teste de Mann Whitney, quando da distribuição não paramétrica. O nível de significância utilizado para os testes foi de $\mathrm{p}<0,05$.

\section{Resultados}

Do total de 100 mulheres entrevistadas, $11 \%$ relataram ter provocado aborto e $89 \%$ relataram ter sofrido aborto espontâneo. Foi realizada análise quantitativa dos aspectos associados com o aborto provocado. A Tabela 2 apresenta as variáveis do contexto social e econômico em que estas mulheres estavam inseridas. Variáveis como escolaridade, ocupação, renda familiar e religião formam o panorama descritivo desta análise, com os respectivos indicadores de significância para as diferenças. Quanto à educação formal, as mulheres do grupo com aborto provocado, em relação ao grupo com aborto espontâneo, apresentaram menor escolaridade, sendo mais frequente o nível fundamental (82 versus $36 \%, \mathrm{p}=0,04$ ); menor renda familiar (mediana, $\mathrm{R} \$ 1.000,00$ versus $\mathrm{R} \$ 1.400,00, \mathrm{p}=0,04)$; menor renda pessoal (mediana, $R \$ 200,00$ versus $R \$ 333,00, p=0,04$ ).

Tabela 1. Características das mulheres entrevistadas de acordo com o tipo de aborto

\begin{tabular}{|c|c|c|c|c|c|}
\hline & \multicolumn{2}{|c|}{$\begin{array}{c}\text { Espontâneo } \\
(\mathrm{n}=22)\end{array}$} & \multicolumn{2}{|c|}{$\begin{array}{c}\text { Provocado } \\
(n=11)\end{array}$} & Valor $p$ \\
\hline \multicolumn{6}{|l|}{ Idade } \\
\hline Mediana (mín-máx) & 26 & $(17-45)$ & 28 & $(17-37)$ & 0,6 \\
\hline \multicolumn{6}{|l|}{ Estado marital (n e \%) } \\
\hline Com companheiro & 19 & 86,4 & 9 & $(81,8)$ & \multirow{2}{*}{$>0,9$} \\
\hline Sem companheiro & 3 & 13,6 & 2 & $(18,2)$ & \\
\hline \multicolumn{6}{|c|}{ Idade gestacional do aborto } \\
\hline Mediana (mín-máx) & 11 & $(7-20)$ & 11 & $(7-15)$ & 0,8 \\
\hline \multicolumn{6}{|l|}{ Paridade (n e \%) } \\
\hline 0 & 4 & 18,2 & 3 & 27,3 & \multirow{2}{*}{0,6} \\
\hline $100+$ & 18 & 81,8 & 8 & 72,7 & \\
\hline \multicolumn{6}{|c|}{ Aborto provocado anterior (n e \%) } \\
\hline Não & 20 & 90,9 & 10 & 90,9 & \multirow{2}{*}{$>0,9$} \\
\hline Sim & 2 & 9,1 & 1 & 9,1 & \\
\hline \multicolumn{6}{|l|}{ Filho vivo (n e \%) } \\
\hline 0 & 4 & 18,2 & 3 & 27,3 & \multirow{2}{*}{0,6} \\
\hline $100+$ & 18 & 81,8 & 8 & 72,7 & \\
\hline
\end{tabular}


Tabela 2. Características socioeconômicas das mulheres de acordo com o tipo de aborto

\begin{tabular}{|c|c|c|c|c|c|}
\hline \multirow[b]{2}{*}{ Escolaridade (n e \%) } & \multicolumn{2}{|c|}{$\begin{array}{c}\text { Espontâneo } \\
n=22\end{array}$} & \multicolumn{2}{|c|}{$\begin{array}{c}\text { Provocado } \\
n=11\end{array}$} & \multirow[t]{2}{*}{ Valor $p$} \\
\hline & & & & & \\
\hline Fundamental & 8 & 36,4 & 9 & 81,8 & \multirow{2}{*}{0,03} \\
\hline Médio/superior & 14 & 63,6 & 2 & 18,2 & \\
\hline \multicolumn{6}{|l|}{ Ocupação (n e \%) } \\
\hline Com atividade laboral & 19 & 86,4 & 7 & 63,6 & \multirow{3}{*}{0,2} \\
\hline Sem atividade laboral & 1 & 4,5 & 2 & 18,2 & \\
\hline Estudante & 2 & 9,1 & 2 & 18,2 & \\
\hline \multicolumn{6}{|l|}{ Renda familiar, reais } \\
\hline Mediana (mín-máx) & 1400 & $(600-2.000)$ & 1000 & $(500-1.400)$ & 0,04 \\
\hline \multicolumn{6}{|l|}{ Renda per capita, reais } \\
\hline Mediana (mín-máx) & 333 & $(130-450)$ & 200 & $(150-433)$ & 0,03 \\
\hline \multicolumn{6}{|l|}{ Religião (n e \%) } \\
\hline Católica & 11 & 50,0 & 8 & 72,7 & \multirow{4}{*}{0,004} \\
\hline Evangélica & 9 & 40,9 & 0 & - & \\
\hline Outras & 0 & - & 3 & 27,3 & \\
\hline Sem religiã̃o & 2 & 9,1 & 0 & - & \\
\hline
\end{tabular}

Quanto à distribuição das mulheres de acordo com a religião, verificou-se diferença significativa $(p=0,004)$ nas que relataram religião evangélica, de forma que não houve caso de aborto provocado nesse grupo.

Quando analisados os aspectos emocionais relatados na vivência do aborto, seja provocado ou espontâneo, buscou-se categorizar os sentimentos em positivos, negativos e ambivalentes para a mulher em momentos específicos do processo de aborto. No grupo com aborto provocado, quando comparado com o grupo com aborto espontâneo, houve maior frequência de sentimentos negativos na suspeita $(81,8$ versus $22,8 \%, p=0,004)$ e na confirmação ( 72 versus $22 \%, p=0,03)$ da gravidez. Não houve diferença quanto à ocorrência de sentimentos ambivalentes na suspeita da gravidez na comparação entre os grupos $(9,1$ versus 9,1\%). Na análise de conteúdo quanto aos sentimentos relatados na confirmação da gravidez, no grupo com aborto provocado, a ocorrência de sentimentos negativos foi significativamente maior $(72,8 \%)$ quando comparado ao grupo com aborto espontâneo $(22,8 \%, p=0,029)$. Não se constatou diferença significativa quanto à ocorrência de sentimentos de culpa na comparação entre os grupos com aborto espontâneo e provocado (22,7 versus $54,5 \%$, $\mathrm{p}=0,117)$. Também não foi constatada diferença entre os grupos com aborto espontâneo e provocado quando a mulher foi questionada sobre a falta de apoio por parte de familiares ou pelo parceiro $(36,4$ versus $27,3 \%, \mathrm{p}=0,709)$.

\section{Discussão}

O presente estudo demonstra que, entre mulheres que procuram atendimento em hospitais da periferia da cidade de São Paulo, o aborto provocado está relacionado a condições socioeconômicas desfavoráveis. Além disso, a experiência na suspeita e confirmação da gravidez é pautada por sentimentos negativos nos casos de aborto provocado.

Neste estudo, as mulheres do grupo com aborto provocado apresentaram escolaridade e renda familiar significativamente inferiores em comparação ao grupo de aborto espontâneo. Outros trabalhos são conflitantes, pois em um deles não foi observada associação entre menor grau de escolaridade e relato de aborto induzido ${ }^{14}$, mas em outros dois se observou esta correlação ${ }^{15,16}$. Nesse sentido, duas ressalvas se fazem necessárias. A primeira refere-se ao fato apontado pelos estudos de que a escolaridade associa-se negativamente à gravidez indesejada e positivamente à prática de aborto, ou seja, mulheres com maior escolaridade têm menores chances de gravidez indesejada, mas, uma vez grávidas, têm maiores chances de interromper uma gestação do que mulheres de menor escolaridade ${ }^{17-19}$. Além disso, a maioria das pesquisas sobre aborto é desenvolvida em hospitais públicos, e a mulher com melhor nível socioeconômico utiliza serviços privados de saúde. Portanto, ao analisar a ocorrência de aborto ao longo da vida, é fundamental levar em conta que tal oposição pode mascarar o efeito final da escolaridade na sua prática. $\mathrm{O}$ que apreende de fato é que os contextos socioeconômicos e culturais podem ser mais determinantes nas escolhas reprodutivas, pois mulheres com nível socioeconômico melhor tendem a utilizar recursos privados para realização do aborto, e as mulheres economicamente menos favorecidas tendem a se sujeitar a abortos inseguros, com posterior hospitalização ou não no SUS ${ }^{8,19,20}$.

Um estudo de revisão ${ }^{19}$ mostra que, nas últimas três décadas, emergiram e se consolidaram no Brasil novos campos de produção científica articulando as temáticas de gênero, sexualidade e saúde reprodutiva ${ }^{19}$. O aborto está entre os objetos investigados, reconhecendo-se sua importância como problema de saúde pública no país, buscando-se um diálogo com movimentos sociais nacionais e internacionais que incluem o tema como prioridade em suas agendas.

Este estudo, realizado em dois hospitais públicos da periferia da cidade de São Paulo, revela a condição de exclusão das mulheres entrevistadas. Se o sistema público de saúde vem melhorando, muito ainda há de se fazer para $\mathrm{o}$ atendimento integral das reais necessidades da população, em especial na atenção à saúde da mulher. Mulheres com recursos financeiros têm acesso a métodos seguros de interrupção da gestação, enquanto outras, de classes menos favorecidas, são obrigadas a se submeter a procedimentos inseguros, que podem colocá-las em condições de risco de morte. É relatado que um dos motivos para a mulher recorrer ao aborto refere-se à falta de condições financeiras, seguido pela falta de apoio do companheiro ${ }^{10,11,21,22} . \mathrm{Na}$ presente pesquisa não se constatou diferença a respeito do 
apoio recebido por parte de familiares ou pelo parceiro na comparação dos grupos de aborto provocado e espontâneo.

Portanto, se faz necessária a discussão sobre as razões que levam a mulher a abortar e as consequências dessa decisão. De acordo com Vieira et al. ${ }^{23}$, para saber o que seria o melhor para cada mulher, seria preciso estabelecer relacionamento interpessoal de confiança mútua, e o profissional deve estar atento aos limites de sua atuação, uma vez que pode ferir o princípio da autonomia. Contudo, consideram que os governos devem se esforçar para promover os direitos, os graus de protagonismo social e a saúde da mulher; devem tentar prevenir a gravidez indesejada por meio da educação, do aconselhamento e da disponibilização da informação adequada que permita decisões reprodutivas adequadas, recorrendo ao planejamento familiar, e não ao aborto, que não constitui método contraceptivo.

Diante desses sentimentos, o aborto acontece na maioria das vezes em silêncio, permeado pela culpa, desamparo e solidão, já que a sociedade recrimina o aborto e não existe espaço para que se possa falar sobre este significado ${ }^{24,25}$. No Brasil, a legislação só permite o aborto em poucas exceções: quando não há outro meio de salvar a vida da mulher e nas gestações resultantes de violência sexual. No entanto, outros motivos podem levar a mulher a interromper intencionalmente a gravidez. Geralmente, essa opção envolve argumentos individuais e inter-relacionais com o contexto sociocultural no que esta mulher está inserida. Assim, de maneira geral são fundamentados em questões sociais, econômicas e emocionais, que, por vezes, é permeada também pela violência doméstica ou sexual $^{19,20,24,25}$.

Em conclusão, o aborto provocado em mulheres que procuram atendimento em hospitais da periferia da cidade de São Paulo está relacionado a condições socioeconômicas desfavoráveis, o que prejudica a vivência na suspeita e na confirmação da gravidez.

\section{Agradecimentos}

Ao Ministério de Ciência e Tecnologia/Fundo Setorial de Saúde e do Departamento de Ciência e Tecnologia da Secretaria de Ciência, Tecnologia e Insumos Estratégicos - DECIT/SCTIE - do Ministério da Saúde, por intermédio do Conselho Nacional de Desenvolvimento Científico e Tecnológico - CNPq.

\section{Referências}

1. WHO: recommended definitions, terminology and format for statistical tables related to the perinatal period and use of a new certificate for cause of perinatal deaths. Modifications recommended by FIGO as amended October 14, 1976. Acta Obstet Gynecol Scand. 1977;56(3):247-53.

2. Sedgh $G$, Henshaw S, Singh S, Ahman E, Shah $\mathrm{H}$. Induced abortion: estimated rates and trends worldwide. Lancet. 2007;370(9595): $1338-45$

3. Peres SO, Heilborn ML. Considering and submitting to abortion among young people in the context of legal prohibition: the hidden side of teenage pregnancy. Cad Saude Pública. 2006;22(7): $1411-20$

4. Chaves JHB, Oliveira EM, Bezerra AFS, Camano L, Sun SY, Mattar R. O abortamento incompleto (provocado e espontâneo) em pacientes atendidas em Maternidade do Sistema Único de Saúde. Rev Soc Bras Clín Méd. 2011 ;9(3):189-94.

5. Brasil. Ministério da Saúde. Secretaria de Atenção à Saúde. Departamento de Ações Programáticas Estratégicas. Área Técnica de Saúde da Mulher. Norma técnica: atenção humanizada ao abortamento. Brasília (DF): Ministério da Saúde; 2005.

6. Font-Ribera L, Pérez G, Salvador J, Borrell C. Socioeconomic inequalities in unintended pregnancy and abortion decision. J Urban Health. 2008;85(1):125-35.

7. Brasil. Ministério da Saúde. Secretaria de Ciência, Tecnologia e Insumos Estratégicos. Departamento de Ciência e Tecnologia. 20 anos de pesquisas sobre aborto no Brasil. Brasília (DF): Ministério da Saúde; 2009.
8. Diniz D. Aborto e saúde pública no Brasil. Cad Saude Pública. 2007;23(9): 1992-3.

9. Silva RS. Especulações sobre o papel do aborto provocado no comportamento reprodutivo das jovens brasileiras. Rev Bras Estud Popul. 2002;19(2):249-61.

10. Benute GRG, Nomura RMY, Pereira PP, Lucia MCS, Zugaib M. Abortamento espontâneo e provocado: ansiedade, depressão e culpa. Rev Assoc Med Bras. 2009;55(3):322-7.

11. Costa RG, Hardy E, Osis MD, Faundes A. A decisão de abortar: processo e sentimentos envolvidos. Cad Saude Pública. $1995 ; 11(1): 97-105$

12. Grimes DA, Benson J, Singh S, Romero M, Ganatra B, Okonofua $\mathrm{FE}$, et al. Unsafe abortion: the preventable pandemic. Lancet. 2006;368(9550): 1908-19.

13. Mota NP, Burnett M, Sareen J. Associations between abortion, mental disorders, and suicidal behavior in nationally representative sample. Can J Psychiatry. 2010;55(4):239-47.

14. Barbosa RM, Pinho AA, Santos NS, Villela W, Aidar T. Aborto induzido entre mulheres em idade reprodutiva vivendo e não vivendo com HIV/AIDS no Brasil. Ciênc Saúde Coletiva. 2009; 14(4): 1085-99

15. Olinto MTA, Moreira-Filho DC. Fatores de risco e preditores para o aborto induzido: estudo de base populacional. Cad Saude Pública. 2006;22(2):365-75.

16. Kac G, Silveira EA, Oliveira LC, Araújo DMR, Sousa EB. Fatores associados à ocorrência de cesárea e aborto em mulheres 
selecionadas em um centro de saúde no município do Rio de Janeiro, Brasil. Rev Bras Saúde Matern Infant. 2007;7(3):271-80.

17. Sihvo S, Bajos N, Ducot B, Kaminski M. Women's life cycle and abortion decision in unintended pregnancies. J Epidemiol Community Health. 2003;57(8):601-5.

18. Rossier C, Michelot F, Bajos N; COCON Group. Modeling the process leading to abortion: an application to French survey data. Stud Fam Plann. 2007;38(3):163-72.

19. Menezes G, Aquino EML. Pesquisa sobre o aborto no Brasil: avanços e desafios para o campo da saúde coletiva. In: Rocha, MIB \& Barbosa, RM (Orgs). Aborto no Brasil e países do Cone Sul: panorama da situação e dos estudos acadêmicos. Campinas: NEPO-UNICAMP; 2009. p. 119-54.

20. Souza e Silva R, Vieira EM. Frequency and characteristics of induced abortion among married and single women in São Paulo, Brazil. Cad Saude Publica. 2009;25(1):179-87.
21. Diniz D, Medeiros M. Aborto no Brasil: uma pesquisa domiciliar com técnica de urna. Ciênc Saúde Coletiva. 2010;15 Supl 1: 959-66.

22. Adesse L, Monteiro MFG, Levin J. Grave problema de saúde pública e de justiça social. RADIS: Comunicação em Saúde. 2008;(66): 10-5.

23. Vieira LM, Goldberg TBL, Saes SO, Dória AAB. Abortamento na adolescência: um estudo epidemiológico. Ciênc Saúde Coletiva. 2007; 12(5): 1201-8.

24. Diniz NMF, Gesteira SMA, Lopes RLM, Mota RS, Pérez BAG, Gomes NP. Aborto provocado e violência doméstica entre mulheres atendidas em uma maternidade pública de Salvador-BA. Rev Bras Enferm. $2011 ; 64(6): 1010-5$.

25. Nomura RMY, Benute GRG, Azevedo GD, Dutra EMS, Borsari CG, Rebouças MSS, et al. Depressão, aspectos emocionais e sociais na vivência do aborto: comparação entre duas capitais brasileiras. Rev Assoc Med Bras. 2011 ; 57(6):644-50. 